\title{
Numerical Studies on the Influence of Cavity Thermal Expansion on the Performance of a High-Power Gyrotron
}

\author{
Konstantinos A. Avramidis, Andrea Bertinetti, Ferran Albajar, Francesca Cau, Fabio Cismondi, \\ Gerd Gantenbein, Stefan Illy, Zisis C. Ioannidis, John Jelonnek, Senior Member, IEEE, \\ François Legrand, Ioannis Gr. Pagonakis, Yoann Rozier, Tomasz Rzesnicki, Laura Savoldi, Member, \\ IEEE, Manfred Thumm, Fellow, IEEE, Roberto Zanino, Senior Member, IEEE
}

\begin{abstract}
An iterative procedure is described, which models the influence of the thermal expansion of the gyrotron cavity on the expected gyrotron performance. It is a multi-physics simulation method, which involves electrodynamic, thermalhydraulic, and thermo-mechanical simulations. The method is applied to the first European $170 \mathrm{GHz}, 1 \mathrm{MW}$ Continuous Wave prototype gyrotron for ITER. According to the simulations, a performance reduction of $\sim \mathbf{1 5 \%}$ is expected at nominal operating parameters, because of the thermal expansion of the cavity. Alternative operating points to mitigate this effect are proposed and numerically validated. The numerical results are discussed in the light of experimental findings.
\end{abstract}

Index Terms-ECRH, Gyrotron, ITER, Multi-Physics simulation, Thermal expansion

\section{INTRODUCTION}

$\mathrm{H}$ IGH-power gyrotrons, intended for electron cyclotron resonance heating and current drive in present and future magnetic confinement fusion reactors, need to operate in long (seconds to hours) pulses delivering MW-level microwave power at frequencies above $100 \mathrm{GHz}$ [1]. In such gyrotrons, the Ohmic loading of the wall of the resonant cavity is large

Manuscript received October 31, 2017. This work is partly supported by Fusion for Energy under Grant F4E-GRT-553 to the European Gyrotron Consortium (EGYC). EGYC is a collaboration among SPC, Switzerland; KIT, Germany; HELLAS, Greece; IFP-CNR, Italy. The views expressed in this publication are the sole responsibility of the author and do not necessarily reflect the views of F4E and the European Commission. Part of the simulations was performed on the EUROfusion High Performance Computer (Marconi-Fusion).

K. A. Avramidis, G. Gantenbein, S. Illy, Z. C. Ioannidis, I. Gr. Pagonakis, T. Rzesnicki, M. Thumm, and J. Jelonnek are with Karlsruhe Institute of Technology, Institute for Pulsed Power and Microwave Technology, Karlsruhe,76131 Germany. (e-mail: konstantinos.avramidis@ kit.edu).

A. Bertinetti, L. Savoldi, and R. Zanino are with Politecnico di Torino, Dipartimento Energia, NEMO group, Torino, 10129 Italy.

F. Albajar, F. Cau are with Fusion for Energy, Barcelona, 08019 Spain.

F. Cismondi was with Fusion for Energy, Barcelona, 08019 Spain. He is now with EUROfusion Consortium, Garching, 85748 Germany.

F. Legrand is with Thales Electron Devices, Vélizy-Villacoublay, 78140 France.

Y. Rozier was with Thales Electron Devices, Vélizy-Villacoublay, 78140 France. $\left(\sim 1.5-2 \mathrm{~kW} / \mathrm{cm}^{2}\right.$, or even higher) and this has always been one of the major technological limiting factors, as far as output power and efficiency are concerned. Consequently, special attention should be given to the cavity cooling system, to make sure that it is able to handle the corresponding high heat fluxes. In support of this, the modeling of the thermal expansion of the gyrotron cavity during long-pulse operation is of primary importance for assessing the capabilities of the cooling system, for predicting the gyrotron performance, and for interpreting the experimental results.

For accurate modeling of the gyrotron operation in the presence of cavity thermal expansion, a multi-physics simulation combining three fundamental elements is necessary: Electrodynamic modeling of the interaction of the electron beam with the electromagnetic field in the cavity should determine the Ohmic wall loading and the corresponding heat flux to the cavity wall. Then, thermal-hydraulic modeling is needed to calculate the temperature field, taking into account the given heat flux and the configuration of the cavity cooling system. Finally, thermo-mechanical modeling must be used to determine the cavity thermal deformation for the obtained temperature field. The described procedure is iterative: The beam-field interaction needs to be recalculated for the updated cavity deformation and temperature distribution, the new heat flux should be determined, and so on, until convergence to a steady state is reached.

A similar iterative procedure was presented in [2], [3] and applied to the $140 \mathrm{GHz}, 1 \mathrm{MW}$ gyrotron for the stellarator W7-X [4]. However, in those studies the thermal-hydraulic modeling was omitted and an estimated heat transfer coefficient was used instead. The full multi-physics modelling described above, i.e. including thermal-hydraulic calculations, was presented and used in [5] to study a proposed cavity design for a $170 \mathrm{GHz}, 1 \mathrm{MW}$ gyrotron, where the cooling was achieved by mini-channels.

In the present paper, the iterative multi-physics modeling of [5] is applied to the existing European $170 \mathrm{GHz}, 1 \mathrm{MW}$ Continuous Wave (CW) prototype gyrotron for ITER [6]-[9], in which the cavity cooling configuration is based on a porous cooling structure, rather than mini-channels. Two simulation 
approaches are used. The first is a stationary approach, which focuses on determining directly the final steady state. The second is a transient approach, which attempts to emulate the real sequence of events in time until a steady state is reached. It is shown that both approaches yield the same final steady state result for long-pulse operation. This validates further the results obtained in [5], where only the stationary approach was used.

The main focus of the present paper is on assessing the performance of the European $170 \mathrm{GHz}, 1 \mathrm{MW} \mathrm{CW}$ gyrotron for ITER in the presence of cavity thermal expansion, as far as power, efficiency, frequency shift, and mode competition are concerned. It is found that significant performance degradation due to the thermal expansion is to be expected at the nominal operating point. These results, summarized in [10] but also presented here in a more detailed and elaborate manner, have motivated extensive investigations on alternative operating points to mitigate the performance degradation. The present paper further extends [10] by reporting details on those investigations, as well as on the numerical validation of the proposed alternative operating points. In addition, the simulation results are discussed in the light of the experimental findings with the $170 \mathrm{GHz}, 1 \mathrm{MW} \mathrm{CW}$ gyrotron.

The paper is organized as follows: In Section II, an overview of the multi-physics modeling as well as details on the transient and stationary approaches are given. In Section III, the simulation results for the nominal operating point are presented and discussed. In Section IV, the investigations on alternative operating points are reported and the validation of the most promising points is presented. Section V discusses the numerical results and co-relates them to the experimental findings. Section VI gives the summary of this work.

\section{CAVITY STRUCTURE AND MULTI-PHYSICS MODELING}

The schematic of the inner contour of the cylindrically symmetric cavity wall of the European $170 \mathrm{GHz}, 1 \mathrm{MW} \mathrm{CW}$ gyrotron for ITER is shown in Fig. 1. The expected Ohmic wall loading (heat flux) at nominal operating parameters,

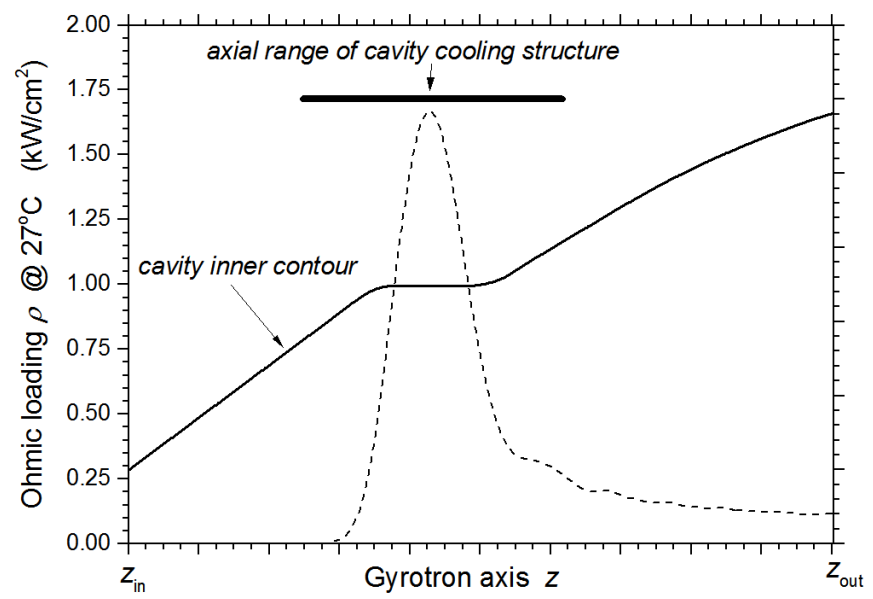

Fig. 1. Schematic of the inner contour of the cavity wall of the European $170 \mathrm{GHz}, 1 \mathrm{MW} \mathrm{CW}$ gyrotron for ITER (thin solid curve). The nominal Ohmic wall loading at room temperature is also shown (dashed curve).

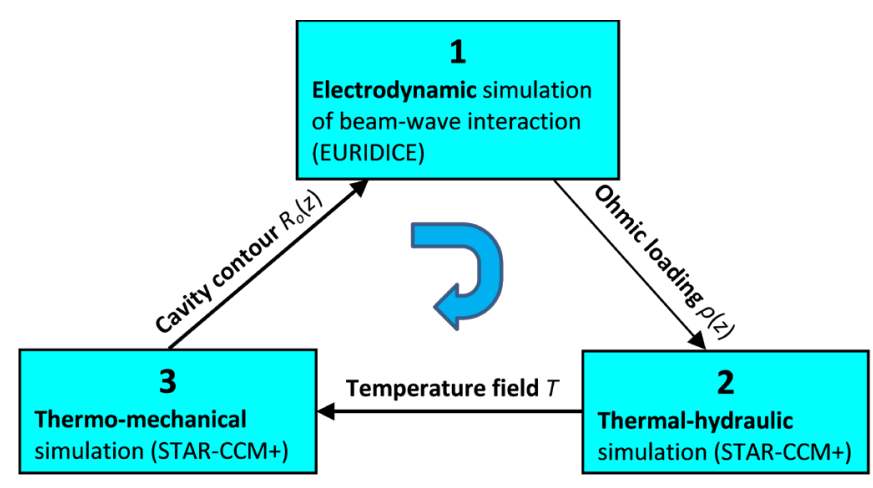

Fig. 2. Iterative multi-physics scheme with three phases per iteration step.

assuming room temperature, is also shown. The cooling system consists of a cooling jacket around the cavity contour with a macro-porous, highly conductive structure at the cavity midsection. This is in order to enhance the cooling at the midsection, since this is the region with the highest loading. The axial range of the porous cooling structure is indicated in Fig. 1 by the horizontal thick line and it is centered on the maximum of the heat flux.

The iterative multi-physics scheme is shown schematically in Fig. 2. The code-package EURIDICE [11] is used for the electrodynamic simulations. At a given operating point, EURIDICE calculates the interaction between the electromagnetic high-frequency field and the electron beam, considering a cavity profile $R_{o}(z)$ along the gyrotron axis $z$, where $R_{o}$ is the distance of the cavity inner surface from the gyrotron axis. The Ohmic wall loading $\rho$ is calculated from the well-known formula [12]:

$\rho=\frac{1}{2 \sigma \delta}\left|\mathbf{H}_{t}\right|^{2}$

Here, $\mathbf{H}_{t}$ is the component of the high-frequency magnetic field that is tangential to the cavity wall, $\sigma$ is the electrical conductivity of the wall, and $\delta$ is the skin depth. The material of the cavity wall is Glidcop and the dependence of $\sigma$ on the temperature is taken into account according to [13]. Since the temperature varies along the cavity axis, the electrical conductivity is a function of $z$. The dependence of the skin depth $\delta=[2 /(\mu \sigma \omega)]^{1 / 2}$ on the wave angular frequency $\omega$ and on the electrical conductivity is also considered in the calculations. Finally, the result of (1) is corrected by the appropriate multiplying factor provided by the Hammerstad/Bekkadal formula [14]-[15], to account for a wall surface roughness of the order of $0.1 \mu \mathrm{m}$ rms.

The thermal-hydraulic and the thermo-mechanical simulations are performed using STAR-CCM+ v10 [16]. In contrast to previous approaches on the modeling of the cavity cooling, where simplified modeling requiring a pre-defined heat transfer coefficient $h$ for the metal/coolant border is adopted (e.g. [2], [3], [17]), an advanced full 3D model [5] is used here, from which the heat transfer to the coolant is computed self-consistently. This removes the significant uncertainties on the correct choice of $h$ and on its dependence 
on temperature. In particular, for the thermal-hydraulic calculations, the 3D, steady state, incompressible flow model, with the $\kappa-\omega$ SST turbulence closure [18] and "all $y+$ " wall treatment is adopted. The VOF multiphase flow model is chosen, and the Rohsenow model [19], [20] is adopted in case of boiling onset. Temperature-dependent material properties are used. The realistic inlet mass flow rate of $45 \mathrm{l} / \mathrm{min}$ at room temperature and an outlet (gauge) pressure of 0 bar are used as boundary conditions. For the thermo-mechanical simulations, a $3 \mathrm{D}$, steady state, finite-element solid-stress model is used, with linear, isotropic and elastic material properties. The input of the cavity (Fig. 1, $z=z_{\text {in }}$ ) is considered fixed, as it is brazed to the gyrotron structure. The output of the cavity (Fig. 1, $z=z_{\text {out }}$ ) is free to expand axially, as it is brazed to the gyrotron launcher, which is free to move axially. The surface of the cavity inner wall is free to expand radially.

There are three time scales to be observed in the multiphysics simulation: The reaction time of the beam-field interaction (and of the subsequent heat flux) to the cavity deformation is of the order of nanoseconds and it can thus be considered instantaneous. Likewise, the wall deformation adjustment to a given temperature distribution can be also considered instantaneous. On the other hand, the time for the stabilization of the temperature field at a given heat flux is of the order of seconds. With respect to this observation, two different approaches for the iterative procedure can be proposed. In the first, a stationary scheme involving successive equilibria is adopted: at each iteration step, the thermalhydraulic calculation is stopped only after the temperature field is stabilized, i.e. after time $\Delta t \rightarrow \infty$. The second approach is a transient one and is closer to what happens in reality. In particular, the first iteration begins at time $t=t_{0}$ with phase 1 (see Fig. 2), and then proceeds to phase 2. However, the thermal-hydraulic simulation of phase 2 runs only for a finite time period $\Delta t_{1}$ (rather than $\Delta t \rightarrow \infty$ ), up to $t=t_{1}=t_{0}+\Delta t_{1}$. Then, the thermo-mechanical calculation of phase 3 is performed and the second iteration is launched at $t=t_{1}$ with phase 1. In the second iteration, the thermal-hydraulic simulation of phase 2 runs up to $t=t_{2}=t_{1}+\Delta t_{2}$, and so on. The choice of the finite time periods $\Delta t_{1}, \Delta t_{2}, \ldots$ should secure that, between two successive iterations, the cavity deformation remains small enough in order for the change in the beamwave interaction to be also small. In this way, this procedure can better emulate the real sequence of events. It can be said that the stationary approach should be considered valid, if its converged result is the same with that obtained by the transient approach at time $t \rightarrow \infty$.

\section{RESULTS AT THE NOMINAL OPERATING POINT}

There are two nominal operating points for the $170 \mathrm{GHz}$, $1 \mathrm{MW}$ CW prototype for ITER, operating at the $\mathrm{TE}_{32,9}$ mode [7]: The first is the High-Voltage Operating Point (HVOP) with beam voltage $V_{b}=79.5 \mathrm{kV}$, beam current $I_{b}=40 \mathrm{~A}$, magnetic field at the cavity $B_{0}=6.78 \mathrm{~T}$, and electron velocity ratio at the cavity $\alpha=1.3$. The second is the Low-Voltage
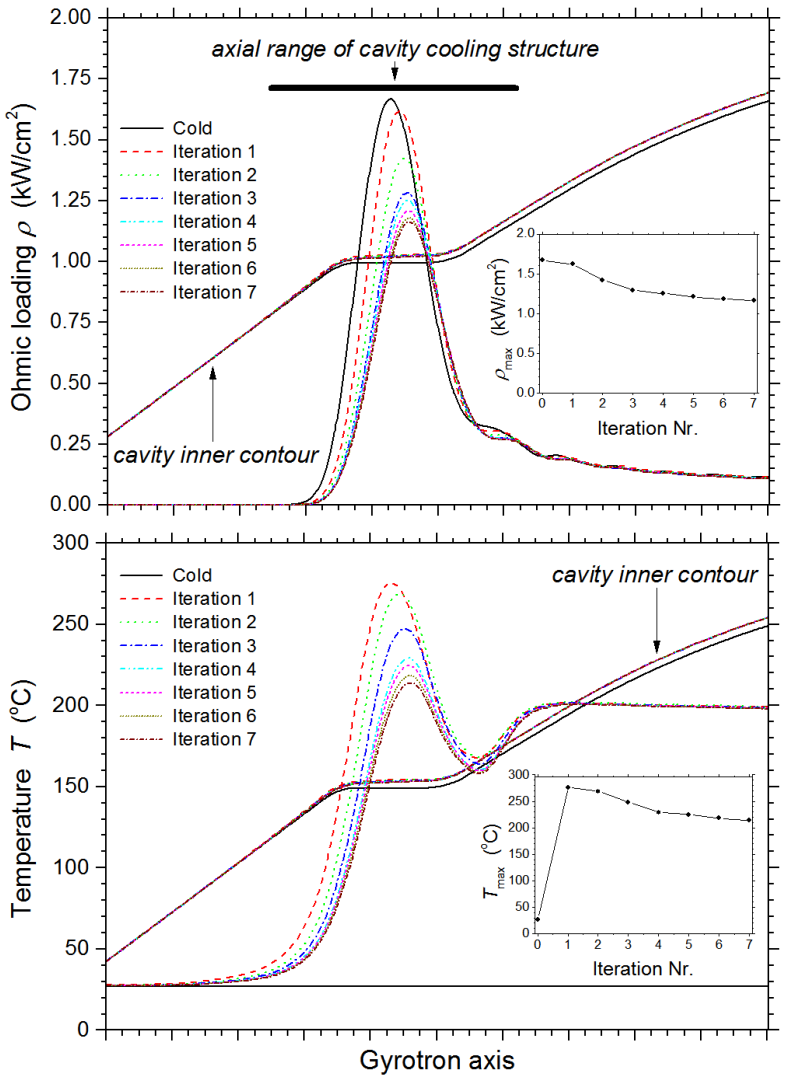

Fig. 3 Stationary modeling of the nominal operating point HVOP: Ohmic wall loading (top) and temperature (bottom) versus cavity axis. Insets show the evolution of the maximum value during iterations.

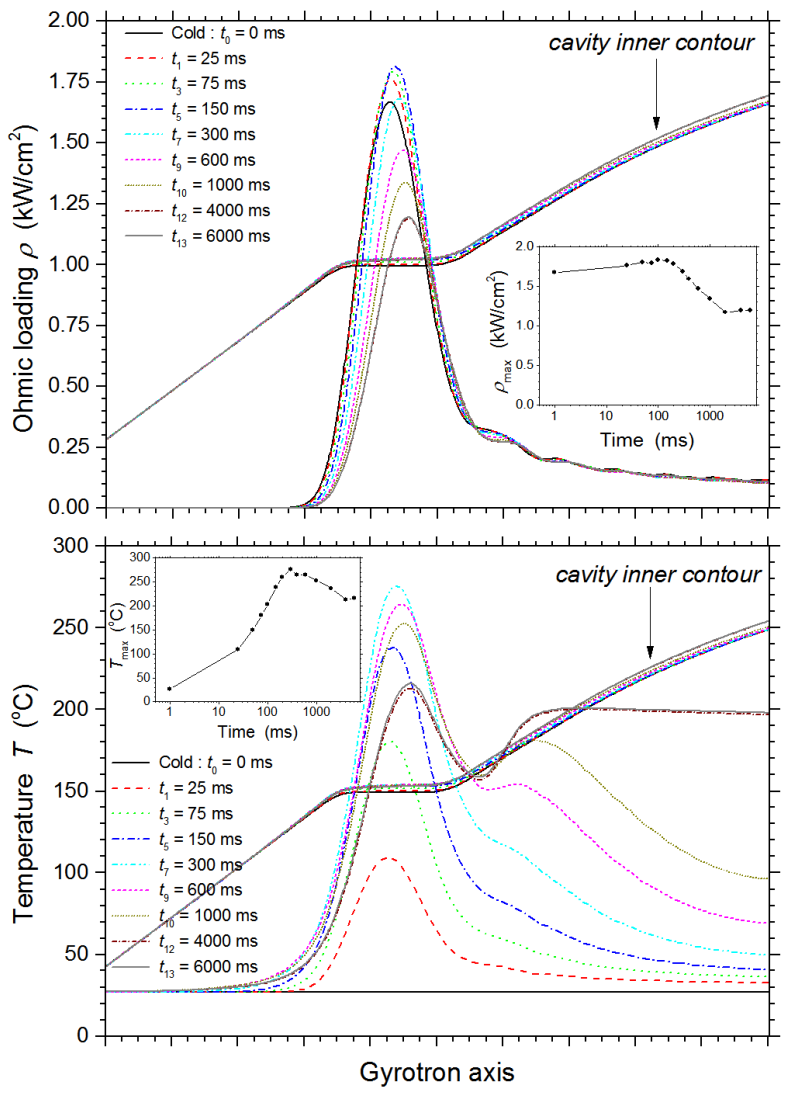

Fig. 4 Transient modeling of the nominal operating point HVOP: Ohmic wall loading (top) and temperature (bottom) versus cavity axis. (For clarity, only selected time steps are shown.) Insets show the evolution of the maximum value with time. 

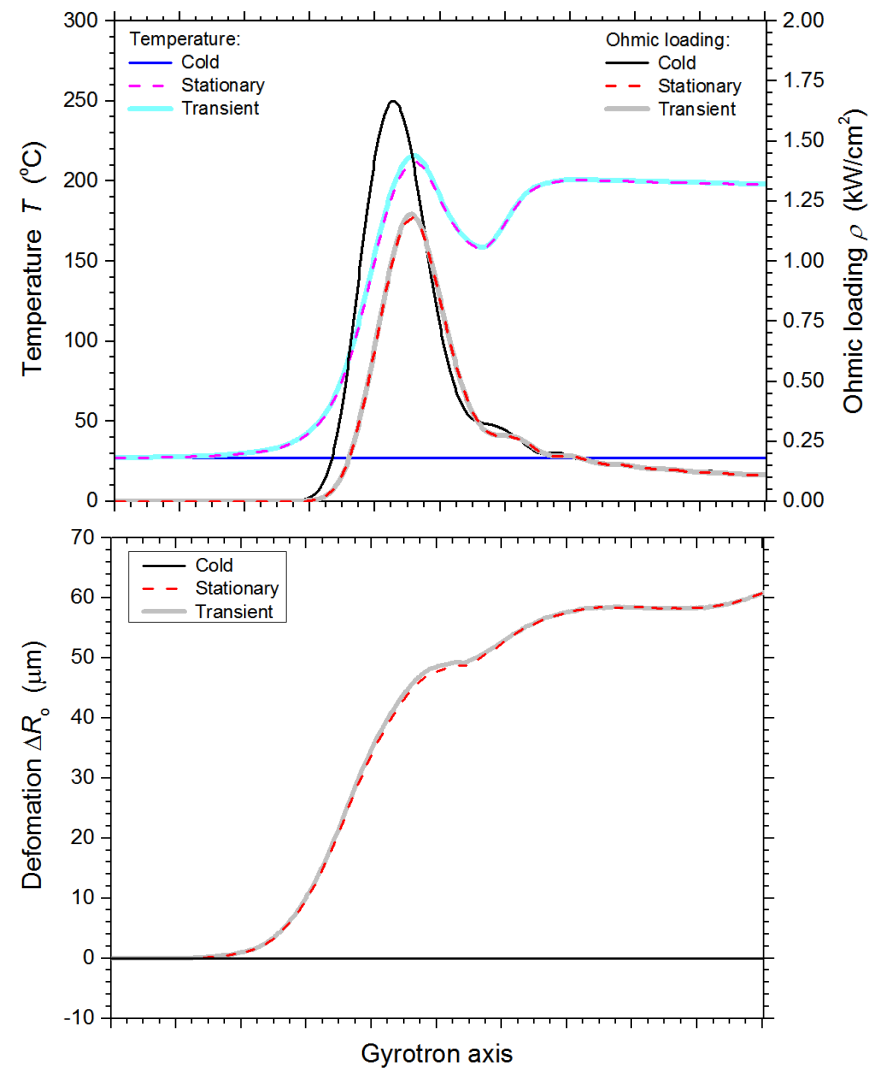

Fig. 5 Comparison of final results on temperature and Ohmic wall loading (top), and on deformation $\Delta R_{\mathrm{o}}=R_{\mathrm{o}, \text { hot }}-R_{\mathrm{o} \text {,cold }}$ of the cavity inner contour (bottom), using the stationary and the transient approach. The results for the non-deformed cavity at room temperature (cold) are also shown.

Operating Point (LVOP) with $V_{b}=71.0 \mathrm{kV}, I_{b}=45 \mathrm{~A}$, $B_{0}=6.69 \mathrm{~T}, \alpha=1.2$. The beam voltage $V_{b}$ is related to the electron kinetic energy $E_{k}=|e| V_{b}$, where $e$ is the electron charge. The multi-physics simulations focused on the HVOP. At the beginning, the beam-wave interaction was simulated by EURIDICE in the non-deformed cavity at room temperature. The resulting Ohmic wall loading is shown in Fig. 1 and it was used in STAR-CCM+ to initiate the iterations.

The stationary approach of successive equilibria was tried first. Each three-phase iteration step takes about $12 \mathrm{~h}$ on a modern multi-core machine. The electromagnetic simulations considered only the operating $\mathrm{TE}_{32,9}$ mode and an ideal electron beam with no spreads in energy, velocity, and guiding center. The results of the iterative procedure are shown in Fig. 3. A convergence, i.e. a consistency (between two successive iterations) of Ohmic loading $\left(\delta \rho_{\max }<1.7 \%\right.$ rel.), temperature field $\left(\delta T_{\max }<1.8 \%\right.$ rel. $)$, and deformation

TABLE I

Calculated Performance at Nominal Operating Point HVOP (SINGLE-MODE, IDEAL E-BEAM)

\begin{tabular}{lll}
\hline \hline & No deformation & $\begin{array}{l}\text { Final deformation } \\
\text { (Stationary / Transient) }\end{array}$ \\
\hline $\begin{array}{l}\text { Power at window } P_{w}(\mathrm{~kW}) \\
\text { Total efficiency } \eta_{\text {tot }}(\%)\end{array}$ & 1053 & $875 / 883$ \\
$\begin{array}{l}\text { w/o depressed collector } \\
\text { Frequency }(\mathrm{GHz})\end{array}$ & 32.5 & $27.0 / 27.3$ \\
$\begin{array}{l}\text { Diffractive } Q \\
\text { w/o elec. beam }\end{array}$ & 170.24 & $169.85 / 169.85$ \\
\hline \hline
\end{tabular}

TABLE II

OPERATING PoINTS AND SimUlated PERformance (MULTI-MODE, REALISTIC E-BEAM)

\begin{tabular}{cccccccccc}
\hline \hline \multirow{2}{*}{ Point } & $\begin{array}{l}V_{b} \\
(\mathrm{kV})\end{array}$ & $\begin{array}{l}I_{b} \\
(\mathrm{~A})\end{array}$ & $\begin{array}{l}B_{0} \\
(\mathrm{~T})\end{array}$ & $\alpha$ & $\begin{array}{l}P_{w} \\
(\mathrm{~kW})\end{array}$ & $\begin{array}{l}\eta_{\mathrm{tot}} \\
(\%)\end{array}$ & $\begin{array}{l}\rho_{\max }{ }^{*} \\
\left(\mathrm{~kW} / \mathrm{cm}^{2}\right)\end{array}$ & $\begin{array}{l}\Delta \mathrm{V} \\
(\mathrm{kV})\end{array}$ \\
OP1 & 78.5 & 40.0 & 6.76 & 1.4 & 1012 & 32.2 & 1.75 & 1.5 \\
OP2 & 72.5 & 45.0 & 6.70 & 1.3 & 1036 & 31.8 & 1.73 & 2.0 \\
OP3 & 70.5 & 45.0 & 6.68 & 1.3 & 1010 & 31.8 & 1.69 & 2.0 \\
\multicolumn{7}{c}{ Nominal operating points } & (non-deformed cavity) & \\
HVOP & 79.5 & 40.0 & 6.78 & 1.3 & 1009 & 31.7 & 1.98 & 2.5 \\
LVOP & 71.0 & 45.3 & 6.69 & 1.2 & 1049 & 32.6 & 1.93 & 2.0 \\
\hline \hline
\end{tabular}

Assuming $T_{\max }=250^{\circ} \mathrm{C}$.

$\left(\delta R_{\mathrm{o}}<1 \mu \mathrm{m}\right)$, was reached after 7 iterations. The simulations were then repeated with the transient approach. The results are shown in Fig. 4. The final steady state, where the temperature field is stabilized, was reached after 13 iterations. Time intervals $\Delta t_{i}$ with a duration ranging from $25 \mathrm{~ms}$ (at the beginning of the transient) to $2 \mathrm{~s}$ (at the end of the transient) have been used. As discussed in section II, in order to secure small changes in the beam-wave interaction the choice of $\Delta t_{i}$ was based on the requirement that the cavity deformation between two successive iterations should be smaller than $10 \mu \mathrm{m}$. (It should be mentioned that this number is of the order of the manufacturing accuracy.)

As it turns out, both approaches result practically in the same final state and, in that respect, they can be considered equivalent. This is clear in Fig. 5, where the final results of the two approaches are compared, and it further validates the results of [5], which were obtained only with the stationary approach. The equivalence of the two approaches is also shown by the calculated gyrotron performance considering the hot, deformed cavity of Fig. 5 after the convergence of the iterative schemes. This performance is summarized in Table I. The total efficiency without depressed collector is defined as $\eta_{\text {tot }}=P_{w} /\left(V_{b} I_{b}\right)$, where $P_{w}$ is the power at the gyrotron window.

Apparently, a $\sim 15 \%$ reduction with respect to the expected performance is predicted. The drop in interaction efficiency is a result of the decrease of the diffractive quality factor $Q$ of the operating $\mathrm{TE}_{32,9}$ mode, because the cavity thermal expansion causes an opening of the cavity towards the output, i.e. a bellshaped deformation (see Fig. 5, bottom). The information provided by the transient approach (Fig. 4) illustrates this effect: Up to $150 \mathrm{~ms}$, the temperature in the cavity midsection is rising much faster than in the cavity output taper, due to the shape of the Ohmic wall loading. In this phase, the deformation of the output taper is small and this does not reduce $Q$. At the same time the Ohmic wall loading is increasing up to $1.82 \mathrm{~kW} / \mathrm{cm}^{2}$, following the decrease of the wall conductivity due to temperature increase. After $150 \mathrm{~ms}$, the increase of the temperature at the output taper causes the bell-shaped deformation decreasing $Q$. The beam-wave interaction becomes less efficient, producing less microwave power and as a result the Ohmic wall loading decreases down to $1.19 \mathrm{~kW} / \mathrm{cm}^{2}$.

The finding of a bell-shaped deformation is in contrast to 

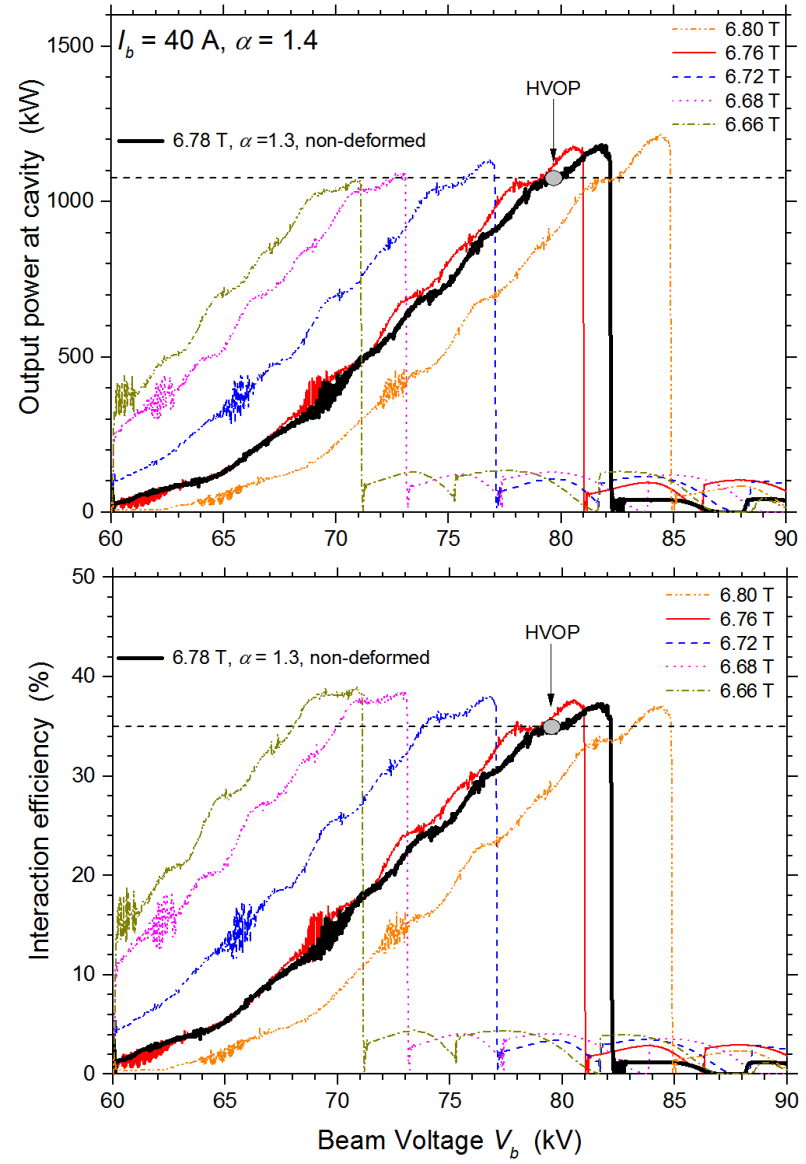

Fig. 6 Typical single-mode parametric sweep: Power $P_{\text {out }}$ exiting the cavity (top) and interaction efficiency $\eta_{\mathrm{el}}$ (bottom) versus the beam voltage at several magnetic field values, assuming a fixed beam current $I_{b}=40 \mathrm{~A}$ and a fixed electron velocity ratio $\alpha=1.4$. The dashed horizontal lines indicate the targeted performance. The thick black curve is related to the nominal operating point HVOP in the non-deformed cavity and is included for comparison.

Note: Assuming 5\% internal losses between cavity output and window we have $P_{w}=0.95 P_{\text {out }}$ and $\eta_{\text {tot }}=0.95 \eta_{\mathrm{el}} /\left(1+Q / Q_{\text {ohm }}\right) . Q_{\text {ohm }}$ is the Ohmic quality factor of the operating mode in the cavity.

the intuitive belief of a barrel-shaped deformation, obtained by observing the profile of the Ohmic wall loading alone. It motivated investigations on alternative operating points to recuperate the performance, as reported in the following section. Although the multi-physics simulations addressed only the nominal operating point HVOP, it is expected that the results and the performance degradation for the nominal point LVOP would be very similar, given the similarity of the Ohmic wall loading profiles and of the interaction efficiencies at the two points.

\section{INVESTIGATIONS ON ALTERNATIVE OPERATING POINTS}

A comprehensive sweep on operating parameters was undertaken, to identify alternative suitable operating points that would recover both power and efficiency at permissible levels of Ohmic wall loading and with good stability margin $\Delta V$ of the operating mode before mode loss. The considered domain of realistically achievable operating values has been the following: $60 \mathrm{kV}<V_{b}<90 \mathrm{kV}, \quad 40 \mathrm{~A}<I_{b}<55 \mathrm{~A}$, $6.62 \mathrm{~T}<B_{0}<6.82 \mathrm{~T}$, and $1.2<\alpha<1.4$. For each set of
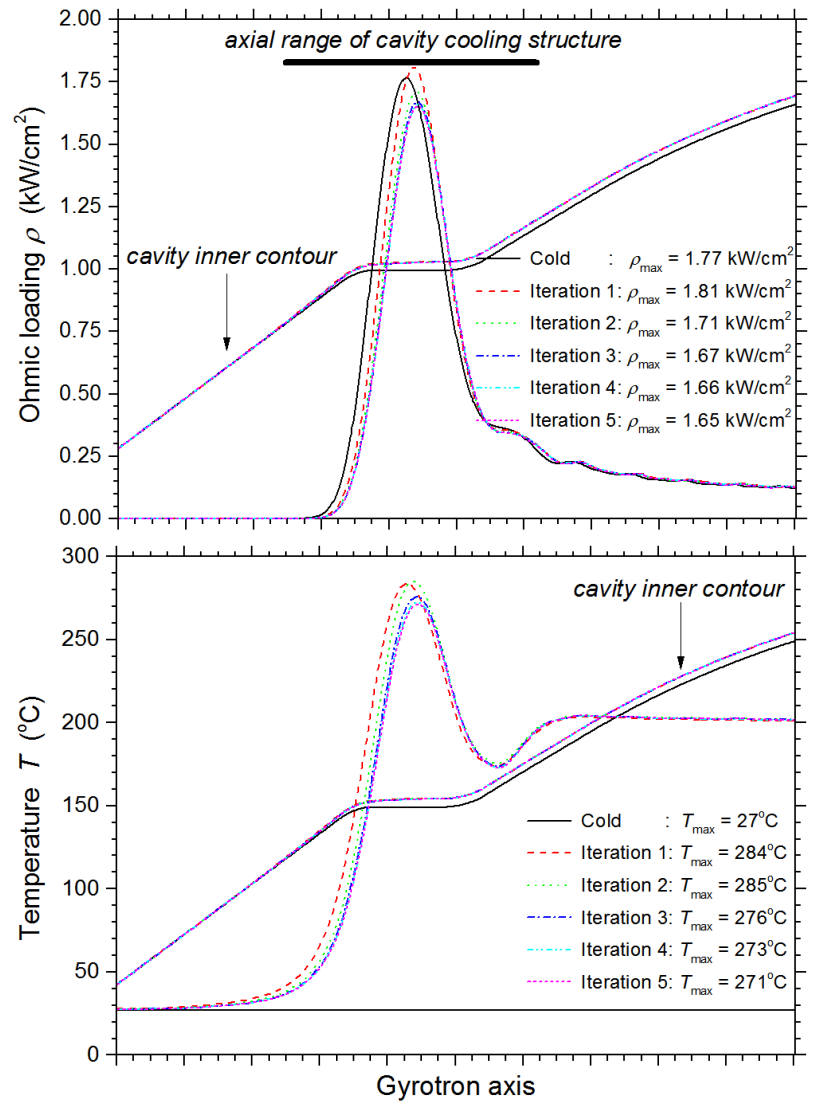

Fig. 7 Stationary modeling of the alternative operating point OP1: Ohmic wall loading (top) and temperature (bottom) versus cavity axis.

parameters, EURIDICE simulated single-mode beam-wave interaction with the operating $\mathrm{TE}_{32,9}$ mode in the deformed cavity of Fig. 5. The electron beam was assumed ideal with no spreads. After these investigations, three representative alternative operating points emerged, which are given in Table II (columns 1-5). The high- and low-voltage nominal operating points are also given for comparison.

To illustrate the criteria of identification of appropriate operating points, a typical result of a parametric sweep is shown in Fig. 6. From this result it can be concluded that, from all the operating points shown, the only suitable point is the point $V_{b}=78.5 \mathrm{kV}, I_{b}=40 \mathrm{~A}, B_{0}=6.76 \mathrm{~T}, \alpha=1.4(\mathrm{OP} 1$ in Table II). This is the only point that practically achieves the targeted performance in the deformed cavity (in terms of both power and efficiency) and, simultaneously, exhibits the same stability margin $\Delta V=2.5 \mathrm{kV}$ (before mode loss) with the nominal operating point at the non-deformed cavity.

After their identification using parametric sweeps, the three

TABLE III

Calculated Performance at OPerating Point OP1 (SINGLE-MODE, IDEAL E-BEAM)

\begin{tabular}{lll}
\hline \hline & No deformation & Final deformation \\
\hline Power at window $P_{w}(\mathrm{~kW})$ & 1126 & 1013 \\
Total efficiency $\eta_{\text {tot }}(\%)$ & 35.9 & 32.3 \\
w/o depressed collector & 170.24 & 169.78 \\
$\begin{array}{l}\text { Frequency }(\mathrm{GHz}) \\
\begin{array}{l}\text { Diffractive } Q \\
\text { w/o elec. beam }\end{array}\end{array}$ & 1009 & 692 \\
\hline \hline
\end{tabular}


TABLE IV

EXPERIMENTAL GYROTRON PERFORMANCE

\begin{tabular}{|c|c|c|c|c|c|c|}
\hline Point & $V_{a c c}(\mathrm{kV})$ & $V_{b}{ }^{*}(\mathrm{kV})$ & $I_{b}(\mathrm{~A})$ & $\alpha^{*}$ & $P_{w}(\mathrm{~kW})$ & $\eta_{\text {tot }}(\%)$ \\
\hline \multicolumn{7}{|c|}{ Experimental points close to $L V O P, B_{0}=6.69 \mathrm{~T}$} \\
\hline 1 & 72.3 & 70.2 & 45.4 & 1.32 & $786 \pm 39$ & $24.7 \pm 1.7$ \\
\hline 2 & 72.8 & 70.7 & 47.5 & 1.28 & $811 \pm 41$ & $24.1 \pm 1.7$ \\
\hline \multicolumn{7}{|c|}{ Experimental points close to $H V O P, B_{0}=6.78 \mathrm{~T}$} \\
\hline 3 & 80.8 & 79.0 & 41.0 & 1.41 & $798 \pm 40$ & $24.6 \pm 1.7$ \\
\hline 4 & 80.3 & 78.4 & 41.0 & 1.48 & $809 \pm 40$ & $25.2 \pm 1.8$ \\
\hline
\end{tabular}

"Calculated numerically.

alternative operating points were further verified by multimode electrodynamic simulations with EURIDICE, assuming a realistic electron beam with $6 \% \mathrm{rms}$ spread in $\alpha, 0.1 \% \mathrm{rms}$ kinetic energy spread, and $0.3 \mathrm{~mm}$ guiding-center spread. The considered deformed cavity was again that of Fig. 5. The results confirmed the suitability of the alternative operating points and are summarized in the last four columns of Table II. Mode competition, however, results in an undesired reduction of the stability range $\Delta V$ of the operating mode at the point OP1. This is again a consequence of the low $Q$.

In all the investigations up to this point, the cavity deformation was taken into account by using the deformed cavity of Fig. 5, obtained by multi-physics simulations. However, this is the calculated deformation assuming operation at the nominal operating point HVOP. In order to validate an alternative operating point, a multi-physics simulation should be performed for that point also. This was done for the point OP1 and the results of the iterative multiphysics procedure are shown in Fig. 7. The stationary approach was used, which converged after 5 iterations. The results on gyrotron performance are shown in Table III, where the recovery of power and efficiency is verified.

\section{EXPERIMENTAL RESULTS AND DISCUSSION}

Experimental results, representative of the best performance achieved in long-pulse operation with the European $170 \mathrm{GHz}$, $1 \mathrm{MW}$ CW prototype gyrotron at KIT [9], are given in Table IV. The acceleration voltage $V_{\text {acc }}$ is the experimentally applied voltage. Because of the space charge of the electron beam, the electron kinetic energy is less than $|e| V_{\text {acc. }}$ Using the code ARIADNE [21], [22], it was possible to estimate that, during long-pulse operation, the expected neutralization of the beam space charge in this gyrotron is of the order of $\sim 70-75 \%$. In this way, the corresponding beam voltage $V_{b}$, appearing in Table IV, was calculated. Moreover, ARIADNE was used to calculate the electron velocity ratio $\alpha$ at all the points of Table IV. The measurement uncertainty on power is $\pm 5 \%$, whereas the measurement uncertainty on voltage and current is $\pm 1 \%$. These values are reflected in the given values for power and efficiency.

The essential difference between the nominal operating points HVOP, LVOP and the proposed alternative operating points $\mathrm{OP} 1, \mathrm{OP} 2, \mathrm{OP} 3$, is that the electron velocity ratio $\alpha$ is higher at the alternative points. This was the only way to recover, in simulation, both power and efficiency in the hot cavity with a bell-shaped deformation. The experimental results of Table IV are in line with this finding, in the sense that the best experimental performance is not achieved at the nominal operating points, but at points where the velocity ratio has values higher than nominal. For instance, the experimental points 1, 2 are close to the nominal LVOP, albeit with $\alpha \sim 1.3$, rather than the nominal $\alpha=1.2$. Likewise, the experimental points 3, 4 are close to the nominal HVOP, albeit with $\alpha>1.4$, rather than the nominal $\alpha=1.3$.

Due to lack of additional experimental time at KIT, further optimization of the operation of the gyrotron was not pursued. This is foreseen to be done in 2018 at the Swiss Plasma Center, EPFL, Lausanne, Switzerland, where the European $170 \mathrm{GHz}, 1 \mathrm{MW} \mathrm{CW}$ prototype is currently installed. The goal is to try reaching the alternative points OP1, OP2, OP3. (In principle, this is more or less equivalent to further increase the beam voltage by $\sim 1 \mathrm{kV}$ at the points of Table IV.) However, it is known that operation at higher velocity ratio is prone to excitation of parasitic modes, mode competition, and electron trapping. This is the reason why such operation was not considered during the initial choice of the nominal operating points. It remains to be seen whether the proposed alternative points are actually reachable in experiment and to what degree they recover the targeted performance.

Another experiment that would verify the calculations of this paper will be the monitoring of the gyrotron output power at nominal operation, starting with millisecond pulses and gradually increasing the pulse-length up to 1-2 seconds. According to the presented results, a significant power drop should be recorded as the pulse-length increases. Of course, such an experiment requires a calorimetric load capable of precise power measurement for a wide range of pulse-lengths. Such a load was not available during the KIT experimental campaign [9], where either a short-pulse $(<10 \mathrm{~ms})$ load or a long-pulse ( $>10 \mathrm{~s}$ ) load was used. However, it is expected that the discussed monitoring of the power will be possible during the foreseen experimental campaign at EPFL.

In any case, the results of present study clearly suggest that possible improvements of the cavity cooling system are worth investigating. Calculations have already shown that cooling by using the mini-channel concept would be much more efficient, securing the targeted performance at nominal operation [5]. An alternative, less involved proposal is the improvement, towards the cavity output, of the porous-structure based cooling system, in order to avoid a bell-shaped deformation. Such an improvement has already been implemented in the dual frequency $84 / 126 \mathrm{GHz}, 1 \mathrm{MW}, 2 \mathrm{~s}$ gyrotron, intended for the upgrade of the TCV tokamak at SPC, EPFL, Lausanne [23]. The delivery of this gyrotron is expected within 2017.

\section{SUMMARY}

A multi-physics model has been described, in order to address the influence of cavity thermal expansion on the performance of a high-power gyrotron. The model has been applied to the European $170 \mathrm{GHz}, 1 \mathrm{MW} \mathrm{CW}$ gyrotron for 
ITER. A stationary and a transient modeling approach have been used and their equivalence, with respect to the identification of the final steady state, has been demonstrated. Multi-physics simulations of the nominal long-pulse operation of the gyrotron showed a bell-shaped cavity deformation. This is in contrast to the intuitively expected barrel-shape deformation and it results in a $15 \%$ performance degradation, due to a reduction of the diffractive quality factor of the operating mode. The experimental results up to now have indeed shown a reduced performance at the nominal operating points, in line with the theoretical findings, at least qualitatively. Parametric investigations on alternative operating points to recover gyrotron performance identified several suitable points. Those alternative points have been further verified with multi-mode interaction simulations involving a realistic electron beam, and with multi-physics simulation of the cavity expansion. Further verification of the presented calculations with respect to the alternative operating points is foreseen within the next long-pulse experimental campaign with the $170 \mathrm{GHz}, 1 \mathrm{MW} \mathrm{CW}$ gyrotron.

\section{REFERENCES}

[1] M. Thumm, "Recent advances in the worldwide fusion gyrotron development”, IEEE Trans. Plasma Sci., vol. 42, p. 590, Mar. 2014.

[2] M. Cordova, "Thermo-mechanical study of the cavity of the $1 \mathrm{MW}-$ $140 \mathrm{GHz}$ gyrotron for W7-X," M.S. thesis, IHM, KIT, Karslruhe, Germany, 2013.

[3] M. Cordova, K. Avramidis, S. Illy, J. Jelonnek, C. Wu, "Thermomechanical study of the cavity deformation in the case of the $1 \mathrm{MW}$, $140 \mathrm{GHz} \mathrm{CW}$ gyrotron for W7-X", presented at the Workshop on RF Heating Technology of Fusion Plasmas, Speyer, Germany, Sep. 9-11, 2013.

[4] M. Thumm, S. Alberti, A. Arnold, P. Brand, H. Braune, G. Dammertz, V. Erckmann, G. Gantenbein, E. Giguet, R. Heidinger, J.-P. Hogge, S. Illy, W. Kasparek, H. P. Laqua, F. Legrand, W. Leonhardt, C. Liévin, G. Michel, G. Neffe, B. Piosczyk, M. Schmid, K. Schwörer, M. Q. Tran, "EU Megawatt-class 140-GHz CW gyrotron", IEEE Trans. Plasma Sci, vol. 35, no. 2, pp. 143 - 153, Apr. 2007. DOI: 10.1109/TPS.2007.892144.

[5] A. Bertinetti, K. A. Avramidis, F. Albajar, F.Cau, F. Cismondi, Y. Rozier, L. Savoldi, R. Zanino, "Multi-physics analysis of a $1 \mathrm{MW}$ gyrotron cavity cooled by mini-channels", Fusion Engineering and Design, May 2017, [Online] Available:

https://doi.org/10.1016/j.fusengdes.2017.05.016.

[6] Y. Rozier, F. Albajar, S. Alberti, K. A. Avramidis, T. Bonicelli, F. Cismondi, P.-E. Frigot, G. Gantenbein, V. Hermann, J.-P. Hogge, J. Jelonnek, J. Jin, F. Legrand, G. Lietaer, I. Gr. Pagonakis, T. Rzesnicki, M. Thumm, "Manufacturing and tests of the European $1 \mathrm{MW}, 170 \mathrm{GHz}$ CW gyrotron prototype for ITER", 17th IEEE International Vacuum Electronics Conference (IVEC 2016), 19-21 Apr. 2016, Monterey, CA, USA. DOI: 10.1109/IVEC.2016.7561795.

[7] T. Rzesnicki, F. Albajar, S. Alberti, K. A. Avramidis, W. Bin, T. Bonicelli, F. Braunmueller, A. Bruschi, J. Chelis, P.-E. Frigot, G. Gantenbein, V. Hermann, J.-P. Hogge, S. Illy, Z. C. Ioannidis, J. Jin, J. Jelonnek, W. Kasparek, G. P. Latsas, C. Lechte, M. Lontano, T. Kobarg, I. G. Pagonakis, Y. Rozier, C. Schlatter, M. Schmid, I. G. Tigelis, M. Thumm, M. Q. Tran, M. Losert, J. L. Vomvoridis, A. Zisis, "Experimental verification of the European $1 \mathrm{MW}, 170 \mathrm{GHz}$ industrial CW prototype gyrotron for ITER", Fusion Engineering and Design, Feb. 2017, [Online] Available: https://doi.org/10.1016/j.fusengdes.2017.02.021.

[8] Z. C. Ioannidis, T. Rzesnicki, K. Avramidis, G. Gantenbein, S. Illy, J. Jin, T. Kobarg, I. Gr. Pagonakis, M. Schmid, M. Thumm, J. Jelonnek, V. Hermann, Y. Rozier, F. Legrand, S. Alberti, F. Braunmueller, J.-P. Hogge, C. Schlatter, J. Genoud, M. Q. Tran, W. Kasparek, C. Lechte, J.
L. Vomvoridis, J. Chelis, G. P. Latsas, A. Zisis, I. G. Tigelis, A. Bruschi, M. Lontano, F. Albajar, T. Bonicelli, P.-E. Frigot, "First CW experiments with the EU ITER $1 \mathrm{MW}, 170 \mathrm{GHz}$ industrial prototype gyrotron", presented at 18th International Vacuum Electronics Conference (IVEC 2017), London, United Kingdom, 2017.

[9] Z. C. Ioannidis, T. Rzesnicki, F. Albajar, S. Alberti, K. A. Avramidis, W. Bin, T. Bonicelli, A. Bruschi, I. Chelis, P.-E. Frigot, G. Gantenbein, V. Hermann, J.-P. Hogge, S. Illy, J. Jin, J. Jelonnek, W. Kasparek, G. Latsas, C. Lechte, F. Legrand, T. Kobarg, I. Gr. Pagonakis, Y. Rozier, C. Schlatter, M. Schmid, I. G. Tigelis, M. Thumm, M. Q. Tran, A. Zein, and A. Zisis, "CW experiments with the EU 1-MW, 170-GHz industrial prototype gyrotron for ITER at KIT", IEEE Trans. Electron Devices, vol. 64, no. 9, pp. 3885 - 3892, Sep. 2017. doi: 10.1109/TED.2017.2730242.

[10] K. A. Avramidis, G. Gantenbein, Z. C. Ioannidis, I. Gr. Pagonakis, T. Rzesnicki, M. Thumm, J. Jelonnek, A. Bertinetti, L. Savoldi, R. Zanino, F. Albajar, F. Cau, F. Cismondi, Y. Rozier, F. Legrand, "Numerical studies on the influence of cavity thermal expansion on the performance of a high-power gyrotron", presented at $18^{\text {th }}$ International Vacuum Electronics Conference (IVEC 2017), London, United Kingdom, 2017.

[11] K. A. Avramides, I. Gr. Pagonakis, C. T. Iatrou, and J. L. Vomvoridis, "EURIDICE: A code-package for gyrotron interaction simulations and cavity design", EPJ Web of Conf. vol. 32, 04016, Sep. 2012. DOI: http://dx.doi.org/10.1051/epjconf/20123204016.

[12] J. D. Jackson, "Wave guides and resonant cavities," in Classical Electrodynamics, 2nd ed., Wiley, 1975, ch. 8, sec. 8.1, p. 339.

[13] ITER Material Properties Handbook for Glidcop, ITER Document No. S 74 MA 2, File Code: ITER-AK04-3201, Publication Package No.: 4, p. 1.

[14] E. O. Hammerstad and F. Bekkadal, Microstrip Handbook. Trondheim, Norway: Univ. Trondheim, 1975, pp. 4-8.

[15] L. Tsang, H. Braunisch, R. Ding, and X. Gu, IEEE Trans. Adv. Packag. vol. 33, no. 4, pp. 839 - 856, Nov. 2010. DOI: 10.1109/TADVP.2010.2089789.

[16] Star-CCM+ User's Manual 10.06.

[17] M. H. Beringer, S. Kern, and M. Thumm, "Mode selection and coaxial cavity design for a 4-MW $170-\mathrm{GHz}$ gyrotron, including thermal aspects", IEEE Trans. Plasma Sci., vol. 41, no. 4, pp. 853 - 861, Apr. 2017. DOI: 10.1109/TPS.2013.2251870.

[18] F. R. Menter, "Review of the shear-stress transport turbulence model experience from an industrial perspective," Int. J. of Comp. Fluid Dynamics, vol 23 pp. 305-316, 2009.

[19] W. Rohsenow, "A method of correlating heat-transfer data for surface boiling of liquids", Technical report (Massachusetts Institute of Technology, Heat Transfer Laboratory); no. 5, pp. 969-976, Cambridge, Mass.: M.I.T. Division of Industrial Cooperation, 1951. http://hdl.handle.net/1721.1/61431.

[20] D. L. Youchison, M. A. Ulrickson and J. H. Bullock, "Prediction of critical heat flux in water-cooled plasma facing components using computational fluid dynamics", Fusion Science Technology vol. 60, pp. $177-184,2011$

[21] I. Gr. Pagonakis and J. L. Vomvoridis, "The self-consistent 3D trajectory electrostatic code ARIADNE for gyrotron beam tunnel simulation", Joint 29th Int. Conf. on Infrared and Millimeter Waves and 12th Int. Conf. on THz Electronics, Karlsruhe, Germany, 2004, Conf. Digest, pp. 657-658, M. Thumm and W. Wiesbeck, Eds.

[22] I. Gr. Pagonakis, K. Avramidis, S. Illy, P. Kalaria, G. Gantenbein, and J. Jelonnek, "Electron beam simulation in the overall gyrotron geometry", 9th Int. Workshop on Strong microwaves and Terahertz waves: Sources and applications, Nizhny Novgorod, Russia, 2014, Conf. proc. pp. 155156.

[23] S. Alberti, J. Genoud, T. Goodman, J.-P. Hogge, M. Silva, T. M. Tran, M. Q. Tran, K. Avramidis, I. Gr. Pagonakis, J. Jin, S. Illy, G. Gantenbein, J. Jelonnek, M. Thumm, W. Bin, S. Garavaglia, A. Moro, V. Hermann, F. Legrand, Y. Rozier, "Progress on the upgrade of the TCV EC-system with two $1 \mathrm{MW}$ dual-frequency gyrotrons", Presented at 41st Int. Conf. Infrared Millimeter THz Waves, Copenhagen, Denmark, 2016, [Online] Available: https://doi.org/10.1109/IRMMWTHz.2016.7758479. 
Konstantinos A. Avramidis was born in Athens, Greece, in 1971. He received the Dipl.Ing. and Ph.D. degrees from the School of Electrical and Computer Engineering, National Technical University of Athens, in 1998 and 2006, respectively. He joined the Institute for Pulsed Power and Microwave Technology, Karlsruhe Institute for Technology, Karlsruhe, Germany, in 2013. Dr. Avramidis is a member of the Technical Chamber of Greece.

Andrea Bertinetti received the B.Sc. in Energy Engineering in 2013 and M.Sc. degrees in Energy and Nuclear Engineering in 2015 from the Politecnico di Torino, Turin, Italy. He is currently a Ph.D. student at Politecnico di Torino from 2015. His present activities involve the CFD analyses of the ITER gyrotron cavity and the preliminary modeling of DEMO pressure suppression system.

Ferran Albajar received the master's degree in Industrial Engineering in 1997 and the PhD in nuclear engineering in 2001 from the Polytechnic University of Catalonia. He was a researcher at the Commissariat à l'Energie Atomique (Cadarache), at the University of Pavia (Pavia) and at the Polytechnic University of Catalonia (Barcelona) contributing to the modeling of the energy transport in fusion plasmas. He worked in industry on power systems engineering. He joined F4E in 2008, and since 2011 he is a Senior Technical Officer on the ITER heating systems.

Francesca Cau, photograph and biography not available at the time of publication.

Fabio Cismondi graduated as a nuclear engineer at the Polytechnic School of Torino, Italy, and obtained his doctoral degree at CEA Cadarache and University of Toulon and Var, France, in Optics, Image Treatment and Processing. He worked at Karlsruhe Institute of Technology (KIT), Germany, and at Fusion for Energy, Barcelona, Spain. He is presently working for KIT as part of the Programme Management Unit of EUROfusion/PPPT in Garching bei München, Germany, as Responsible Officer for the Breeding Blanket Work-Package and as responsible of In-Vessel Components Integration for the European DEMO.

Gerd Gantenbein received the Dipl. Phys. and the Dr.-Ing. Degrees in electrical engineering from the University of Karlsruhe, Karlsruhe, Germany, in 1988 and 1993, respectively. Since 2005, he has been with the Institute for Pulsed Power and Microwave Technology of the Karlsruhe Institute of Technology, Karlsruhe, where he is the Head of the division High Power Microwave Technology.

Stefan Illy received the Dipl.-Phys. and Dr.rer.nat. degrees in physics from the University of Karlsruhe, Karlsruhe, Germany, in 1993 and 1997,respectively. He has been with the Institute for Pulsed Power and Microwave Technology, Karlsruhe Institute of Technology, Karlsruhe, since 1999, where he currently leads the Gyrotron Interaction Analysis and Components Group.

Zisis C. Ioannidis received the B.S. degree in physics, the M.S. degree in electronics, and the Ph.D. degree from the National and Kapodistrian University of Athens, Athens, Greece, in 2002, 2004, and 2008, respectively. Since 2016, he has been with the Institute for Pulsed Power and Microwave Technology, Karlsruhe Institute of Technology, Karlsruhe, Germany.

John Jelonnek (M'97-SM'15) received the Dipl.-Ing. and the Dr.-Ing. degrees in electrical engineering from the Hamburg University of Technology, Hamburg, Germany, in 1992 and 2000, respectively. He has been a Professor and the Director of the Institute for Pulsed Power and Microwave Technology with the Karlsruhe Institute of Technology, Karlsruhe, Germany, since 2011.

François Legrand, photograph and biography not available at the time of publication.

Ioannis Gr. Pagonakis received the B.S. degree in physics from the University of Crete, Rethimno, Greece, in 1997, and the Ph.D. degree from the School of Electrical and Computer Engineering, National Technical University of Athens, Athens, Greece, in 2005. Since 2010, he has been a Researcher with the Karlsruhe Institute of Technology, Karlsruhe, Germany.

Yoann Rozier, photograph and biography not available at the time of publication.

Tomasz Rzesnicki received the Dipl.-Ing. degree in the electrical engineering from Universität Karlsruhe, Karlsruhe, Germany, and also from Politechnika Gdanska, Gdansk, Poland, in 2002, and the Ph.D. degree from the Karlsruhe Institute of Technology (KIT) in 2007. Since 2003, he has been with the KIT for the development of high-power gyrotrons. In 2012, he became Leader of the Gyrotron Tests and Measurement Techniques Group at the Institute for Pulsed Power and Microwave Technology at KIT.

Laura Savoldi (M'11) received the M.Sc. degree in nuclear engineering and the Ph.D. degree in Energetics from Politecnico di Torino (PoliTo), Italy. She has been Professor of nuclear engineering at Dipartimento Energia, PoliTo, since 2017. Her research interests are in the development, validation, and application of computational tools for the analysis of thermal-hydraulic transients in tokamak both at system-level and at component-level. She is a member of the American Society of Mechanical Engineers (ASME).

Manfred Thumm (SM'94-F'02) received the Dipl.-Phys. and Dr.rer.nat. degrees in physics from the University of Tübingen, Tübingen, Germany, in 1972 and 1976, respectively. In 1990, he became a Full Professor at the University of Karlsruhe, Karlsruhe, Germany. He was a Director of the Institute for Pulsed Power and Microwave Technology, Karlsruhe Institute of Technology, Karlsruhe, from 1999 to 2011. Dr. Thumm is KIT Distinguished Senior Fellow.

Roberto Zanino (M'11-SM'13) received the M.Sc. degree in nuclear engineering and the Ph.D. degree in energy engineering from Politecnico di Torino (PoliTo), Italy, where he has been a Professor of nuclear engineering at Dipartimento Energia since 2000, Head of graduate studies in energy engineering since 2011, coordinator of the Nuclear Engineering Modeling (NEMO) group since 2015, and vice-president of the Interuniversity Consortium for Technological Nuclear Research (CIRTEN) since 2017. Prof. Zanino is also a member of the American Nuclear Society (ANS), of the American Society of Mechanical Engineers (ASME) and of the International Solar Energy Society (ISES). 